\title{
Posição viciosa de cabeça por astiǵmatismo mal corrigido: relato de caso
}

\author{
Abnormal head position caused by incorrect prescription for astigmatism:case report
}

\author{
Flávia Aug'usta Attié de Castro ${ }^{1}$ \\ Maria Lúcia Habib Simão \\ Christine Mae Morello Abbud ${ }^{3}$ \\ Rosália Maria Simões Antunes Foschini ${ }^{4}$ \\ Harley Edison Amaral Bicas ${ }^{5}$
}

\begin{tabular}{|c|}
\hline RESUMO \\
\hline 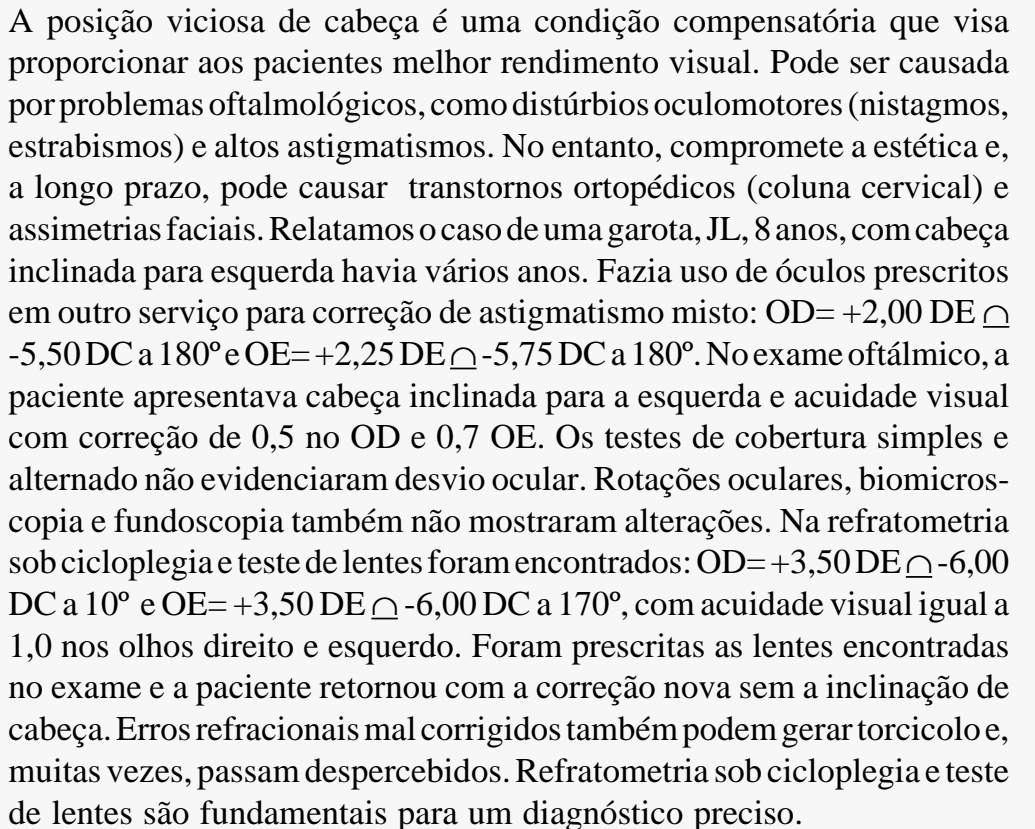 \\
\hline
\end{tabular}

Descritores: Cabeça/fisiopatologia; Postura; Astigmatismo; Estrabismo; Reflexo vestibuloocular/fisiologia; Nistagmo patológico; Músculos oculomotores; Refratometria; Relatos de casos [tipo de publicação]

\section{INTRODUÇ̃̃̃O}

Os termos posição viciosa de cabeça (PVC) e torcicolo são posições persistentes do segmento cefálico em torno dos eixos: vertical ou súperoinferior (rotação para a esquerda ou direita), horizontal transversal ou látero-medial (flexão ou extensão do pescoço) e horizontal longitudinal ou ântero-posterior (inclinação para o ombro esquerdo ou para o direito).

Podem ser secundárias a alterações ortopédicas (como a fibrose congênita do músculo esternocleidomastóideo), neurológicas (como a paralisia supranuclear dos movimentos conjugados e nistagmos) e por problemas oftalmológicos, como distúrbios oculomotores (estrabismo) e altos astigmatismos ${ }^{(1-3)}$. Geralmente, a PVC é uma posição compensatória que visa proporcionar aos pacientes melhor rendimento visual ${ }^{(4-5)}$. No entanto, ela compromete a estética e, a longo prazo, pode causar transtornos ortopédicos (coluna cervical) e assimetrias faciais.

Frente a um paciente com PVC, estrabismo e nistagmo são sempre lembrados como causas, mas altos astigmatismos podem levar o paciente a 
adotar uma posição de cabeça que lhe proporcione melhor acuidade visual.

O presente relato de caso tem como objetivo mostrar um exemplo de PVC secundária a astigmatismo de alto valor, mas com prescrição incorreta e alertar oftalmologistas quanto à importância de um exame refracional bem feito, principalmente em crianças.

\section{RELATO DE CASO}

JL, 8 anos, feminina, foi encaminhada para o setor de Estrabismo do Hospital das Clínicas da Faculdade de Medicina de Ribeirão Preto, Universidade de São Paulo, devido à "cabeça inclinada para o lado esquerdo" havia vários anos (Figura 1). A paciente negou traumatismos anteriores ou doenças sistêmicas. Fazia uso de óculos prescritos em outro serviço para correção de astigmatismo misto: $\mathrm{OD}=+2,00 \mathrm{DE} \subseteq-5,50 \mathrm{DC}$ a $180^{\circ}$ e $\mathrm{OE}=+2,25 \mathrm{DE} \cap-5,75 \mathrm{DC}$ a $180^{\circ}$.

No exame oftalmológico, a paciente efetivamente apresentava PVC inclinada para a esquerda (Figura 2A) e acuidade visual com correção de 0,5 no $\mathrm{OD}$ e 0,7 no $\mathrm{OE}$, mas as lentes dos óculos em uso estavam riscadas. Os testes de cobertura simples e alternado não evidenciaram desvio ocular tanto em posição primária do olhar como nas demais posições (supra, infra, levo e dextroversões). Rotações oculares, biomicroscopia e fundoscopia também não mostraram alterações. Na refratometria sob cicloplegia e teste de lentes foram encontrados:

$$
\begin{aligned}
& \mathrm{OD}=+3,50 \mathrm{DE} \bumpeq-6,00 \mathrm{DC} \text { a } 10^{\circ} \\
& \mathrm{OE}=+3,50 \mathrm{DE} \subseteq-6,00 \mathrm{DC} \text { a } 170^{\circ}
\end{aligned}
$$

com acuidade visual igual a 1,0 nos olhos direito e esquerdo.

Devido à diferença entre as posições dos eixos dos cilindros em uso e as encontradas no exame, em presença de ortoforia, foi feita a hipótese diagnóstica de a PVC ser secundária à posição incorreta dos eixos dos cilindros em uso pela paciente.

Foram prescritas as lentes encontradas no exame e a paciente retornou com a correção nova sem PVC (Figura 2B).

\section{DISCUSSÃO}

Em posição primária do olhar, os eixos dos cilindros negativos encontrados nos óculos antigos estavam no plano horizontal nos dois olhos $\left(0^{\circ}\right.$ ou $\left.180^{\circ}\right)$.

Porém, as posições achadas como corretas para esses eixos eram de $10^{\circ}$ no olho direito (portanto extorcida relativamente à usada) e $170^{\circ}$ no esquerdo (idem); ou, reciprocamente, a posição efetivamente usada do eixo de cada lente achava-se intorcida relativamente à correta, tanto para um olho quanto para o outro. Para melhorar sua visão, com a fixação com o olho esquerdo (o olho dominante), a paciente inclinava a cabeça para a esquerda, posicionando os óculos e o olho esquerdo de maneira que o eixo do cilindro se posicionasse corretamente; a lente esquerda era inclinada no sentido horário, o olho esquerdo realizava movimento compensatório de intorção (anti-horário) e a correção ficava, então, "ajustada" (eixo de $170^{\circ}$ ) (Figura 3).

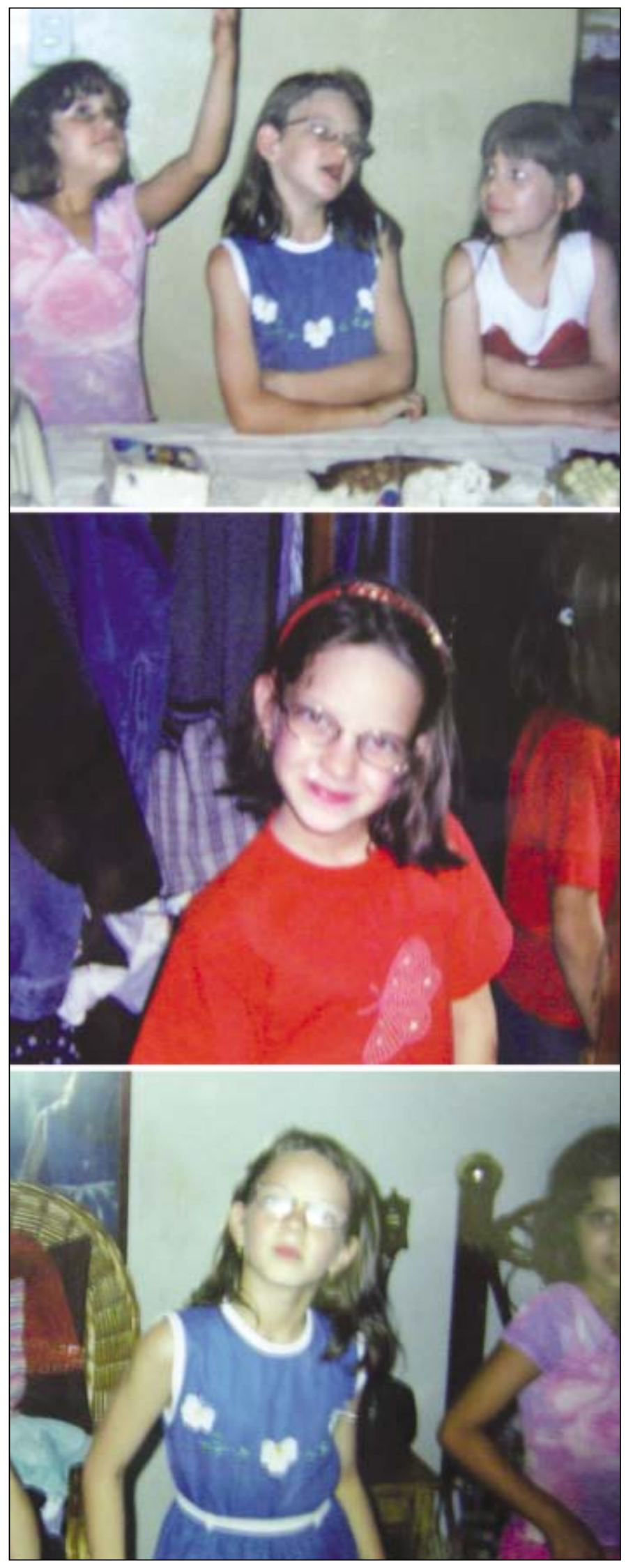

Figura 1 - Fotos prévias da paciente com os óculos antigos 

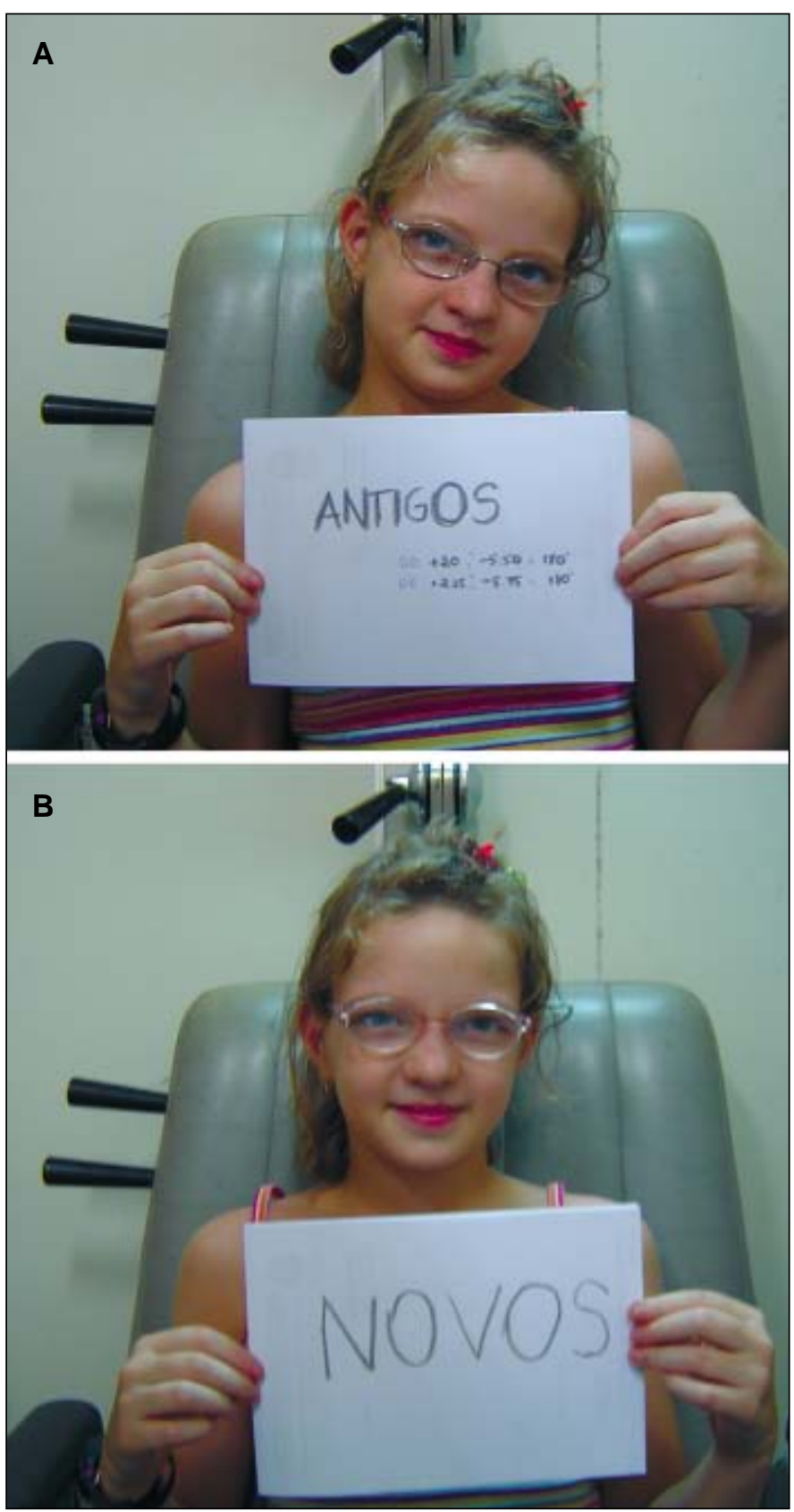

Figura 2 - A: Paciente com os óculos antigos e PVC inclinada para a esquerda; B: Paciente com os óculos novos, sem a PVC

A prescrição incorreta do eixo do cilindro como causa da PVC foi confirmada ao se fazer a oclusão do OE, com os óculos antigos: a paciente inclinou a cabeça para a direita para fixar com o OD, cujo movimento de intorção aproximava o eixo em que se daria a correção de seu astigmatismo ao da posição do eixo da lente cilíndrica usada. Assim, invertia-se a PVC inicial (Figuras 4A e 4B); já com os óculos novos (cilindro prescrito a $10^{\circ}$ no OD), a PVC desapareceu (Figura 2 ).

Os cálculos dos valores dióptricos nas diferentes posições refratométricas para o olho esquerdo e os das respectivas correções possibilitadas pela lente então usada em posição normal

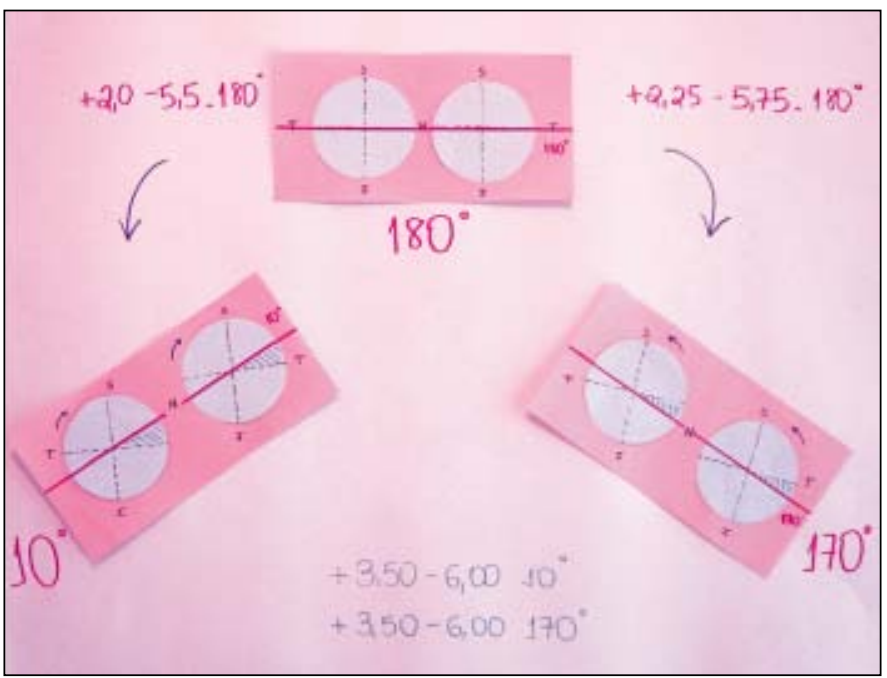

Figura 3 - Esquema representando as mudanças ocorridas nos olhos e lentes dos óculos durante a inclinação da cabeça. Os retângulos rosas representam as lentes dos óculos; os círculos azuis, os olhos, com as posições $\mathbf{S}, \mathrm{I}, \mathrm{T}$ e $\mathbf{N}$ marcadas (superior, inferior, temporal e nasal respectivamente); as linhas vermelhas, marcam o eixo do cilindro nas lentes em uso $\left(180^{\circ}\right)$. Quando há inclinação para a direita, os óculos se inclinam no sentido anti-horário e os olhos no sentido horário (olho direito faz inciclotorção e o esquerdo exciclotorção) e o eixo do cilindro previamente a $180^{\circ}$ fica próximo a $10^{\circ}$ nos dois olhos. Quando há inclinação dos óculos para a esquerda, as lentes fazem movimento horário, os olhos giram no sentido anti-horário (olho direito faz exciclotorção e o esquerdo faz inciclotorção ) e o eixo, antes a $180^{\circ}$ se aproxima a $170^{\circ}$ nos dois olhos

(sem inclinação de cabeça) e com a cabeça compensatoriamente inclinada para a esquerda, mostram que (Figura 4):

a) Para a refratometria $(\mathrm{R})$, o valor dióptrico de menor convergência óptica (+3,50 D) é encontrado na inclinação de $80^{\circ(*)} \mathrm{e}$ o de maior convergência $(-2,50 \mathrm{D})$ na de $170^{\circ}$. Aproximadamente a $30^{\circ}\left(\mathrm{e} 210^{\circ}\right)$ e a $130^{\circ}\left(\mathrm{e} 310^{\circ}\right.$ ) (isto é, mais proximamente ao eixo de $170^{\circ}$ e $350^{\circ}$ que ao de $80^{\circ}$ e $260^{\circ}$ ) o valor dióptrico é nulo.

b) Para a lente usada (+2,25 D sf $\subseteq-5,75 \mathrm{D}$ cil $\left./ 180^{\circ}\right)$ na posição normal da cabeça (S) a maior convergência óptica $(-3,50 \mathrm{D})$ está no eixo de $180^{\circ}$ e a menor $(+2,25 \mathrm{D})$ a $90^{\circ}$. Os valores nulos acham-se aproximadamente nos eixos de $51^{\circ}$ (e $231^{\circ}$ ) e $129^{\circ}$ (e $\left.308^{\circ}\right)$ isto é, mais aproximadamente ao eixo $\left(90^{\circ}\right.$ e $\left.270^{\circ}\right)$ de menor valor absoluto $(+2,25 \mathrm{D})$ que ao seu perpendicular (eixo de 0 a $180^{\circ}$, com valor $-3,50 \mathrm{D}$ ).

c) Para a lente usada na inclinação de cabeça (C), supondose que a torção ocular situe os meridianos oculares nas posições das correções cilíndricas equivalentes, a maior convergência óptica $(-3,50 \mathrm{D})$ passa à inclinação de $170^{\circ}$ (e $\left.350^{\circ}\right)$ e a menor $(+2,25 \mathrm{D})$ à posição de $80^{\circ}\left(\mathrm{e} 260^{\circ}\right)$. Os eixos cujos valores são nulos passam, correspondentemente, aos eixos (aproximados) de $41^{\circ}\left(\mathrm{e} 221^{\circ}\right)$ e $119^{\circ}\left(\mathrm{e} 299^{\circ}\right)$.

\footnotetext{
*): Considera-se como valor dióptrico numa "inclinação" o que corresponde ao efeito dado pela posição dos eixos de lentes cilíndricas. Por exemplo, para a refratometria dada como $+3,50 \mathrm{D}$ sf $\frown-6,00 \mathrm{D}$ cil $/ 170^{\circ}$ (ou equivalentemente $-2,50 \mathrm{D}$ sf $\frown+6,00 \mathrm{D} \mathrm{cil} / 80^{\circ}$ ), os valores de maior e menor refração são respectivamente $-2,50 \mathrm{D}$ a $170^{\circ} \mathrm{e}+3,50 \mathrm{D} \mathrm{a} 80^{\circ}$, que podem ser obtidos por essas lentes cilíndricas. Os meridianos oculares correspondentes a esses defeitos ópticos são perpendiculares a tais eixos.
} 

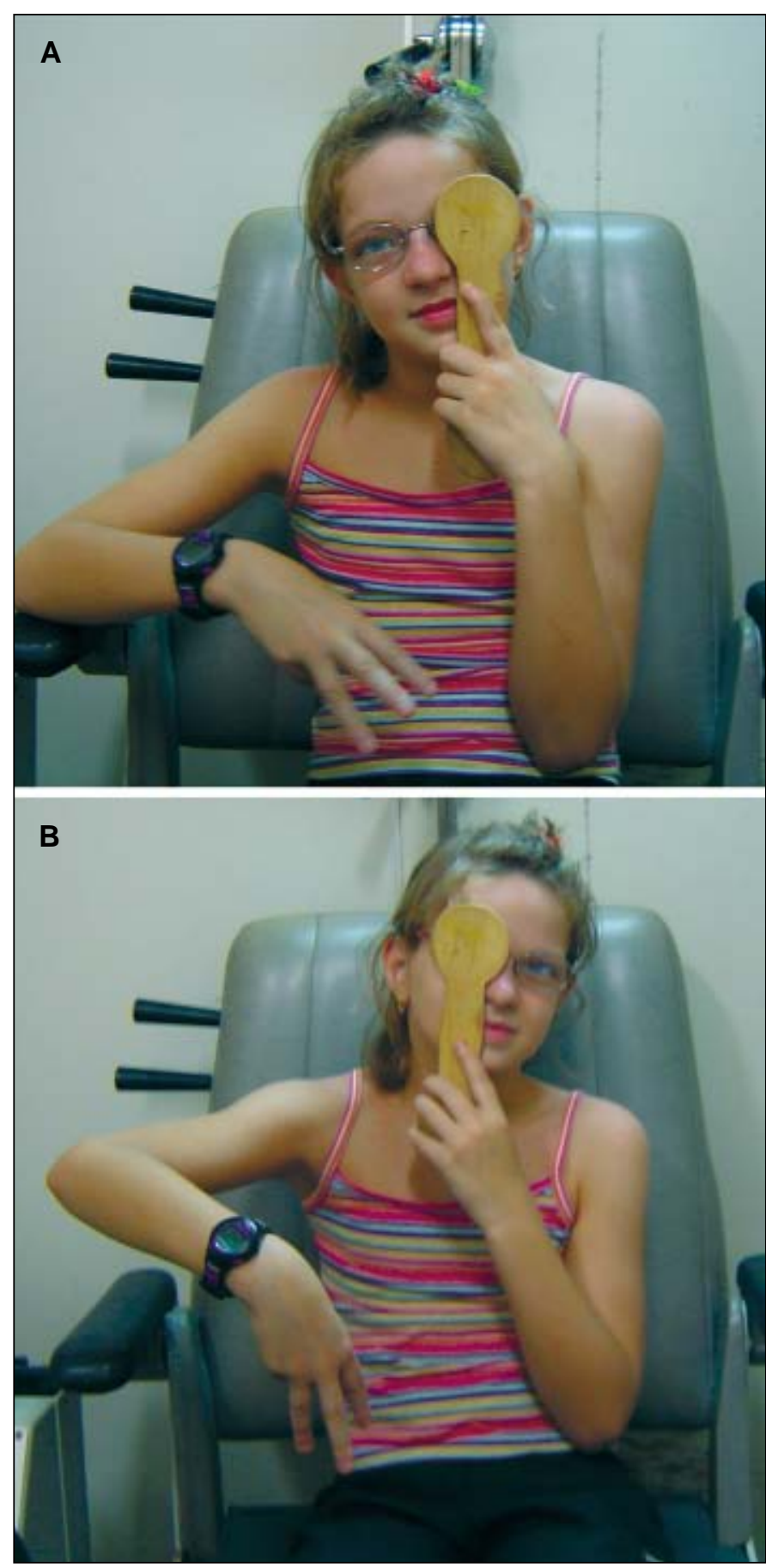

Figura 4 - A: Paciente com os óculos antigos, com olho direito fixando. PVC inclinada para a direita; B: Paciente com os óculos antigos, com olho esquerdo fixando. PVC inclinada para a esquerda

É interessante notar o que ocorre com as diferenças entre o valor refratométrico em cada posição e o efeito da respectiva correção. Com a lente prescrita, usada na posição normal da cabeça, há uma diferença de $10^{\circ}$ entre os valores de maior (ou menor) poder dióptrico da refratometria $(\mathrm{R})$ e da correção $(\mathrm{S})$. Assim, o valor máximo da diferença entre $\mathrm{R}$ e $\mathrm{S}$ é +2,15 D (nos eixos de $43,44^{\circ}$ e $223,44^{\circ}$ ) e o mínimo é $+0,10 \mathrm{D}$ (nos eixos de $133,44^{\circ}$ e $313,44^{\circ}$ ) segundo uma função senoidal.

A figura 5 mostra que a diferença entre as curvas S (valores dióptricos da correção óptica nos diferentes meridianos ou eixos - oculares, com a cabeça ereta) e C (idem, com a cabeça apropriadamente inclinada) é, meramente, de fase. Isto é, a correção óptica dada pela prescrição (S) e que se apresenta assimétrica quando referida à correção ideal (R), é "deslocada" (para a esquerda) por $10^{\circ}$, o que produz uma distribuição mais equilibrada da curva $\mathrm{C}$ relativamente à $\mathrm{R}$. Convém observar que a soma dos erros (diferenças entre $\mathrm{S}$ e $\mathrm{R}$ ou entre $\mathrm{C}$ e $\mathrm{R}$ ) não se altera; ou seja, os espaços entre $\mathrm{S}$ e $\mathrm{R}$ ou entre $\mathrm{C}$ e $\mathrm{R}$ cobrem a mesma área, apenas de modo menos ou mais simétrico (respectivamente nos casos $\mathrm{S}$ e C).

Para uma acomodação de $1,125 \mathrm{D}$, sobraria um astigmatismo misto de $+1,025$ a $-1,025 \mathrm{D}$ nesses eixos. Todavia, se a cabeça for inclinada (C), o eixo da correção cilíndrica passa a coincidir com o valor desejado (cilindro negativo a $170^{\circ} \mathrm{e} \mathrm{ou}$, positivo a $80^{\circ}$ ), restando valores dióptricos de hipermetropia residual $(\mathrm{R}-\mathrm{C}) \mathrm{de}+1,25 \mathrm{D}\left(\mathrm{a} 80^{\circ} \mathrm{e} 260^{\circ}\right) \mathrm{a}+1,00 \mathrm{D}$ (nos eixos de $170^{\circ} \mathrm{e} 350^{\circ}$ ). Com uma acomodação também de $1,125 \mathrm{D}$ sobraria um astigmatismo misto de $+0,125 \mathrm{D}\left(\mathrm{a} 80^{\circ}\right.$ e $260^{\circ}$ ) a $-0,125 \mathrm{D}$ (a $170^{\circ}$ e $350^{\circ}$ ). Ou seja, um ganho de $0,90 \mathrm{D}$ em cada um desses eixos, resultante do astigmatismo remanescente na condição sem a posição viciosa da cabeça $(2,15 \mathrm{D}-0,10 \mathrm{D}=2,05 \mathrm{D})$ contra o que sobra com a posição viciosa de cabeça $(1,25 \mathrm{D}$ $-1,00 \mathrm{D}=0,25 \mathrm{D}$ devida à hipocorreção cilíndrica relativamente à devida pela refratometria).

A grande variação entre os valores máximo e mínimo no caso da correção $\mathrm{S}$ e a pequena variação no caso da correção $\mathrm{C}$, isto é, a correção de um astigmatismo (de 2,05 D a 0,25 D) traduz a preferência por esta última condição. Seria quase o mesmo que comparar qual lente um emétrope preferiria: uma cilíndrica de $-2,00 \mathrm{D}$ (equivalente esférico de $-1,00 \mathrm{D}$ ) produzindo-lhe um

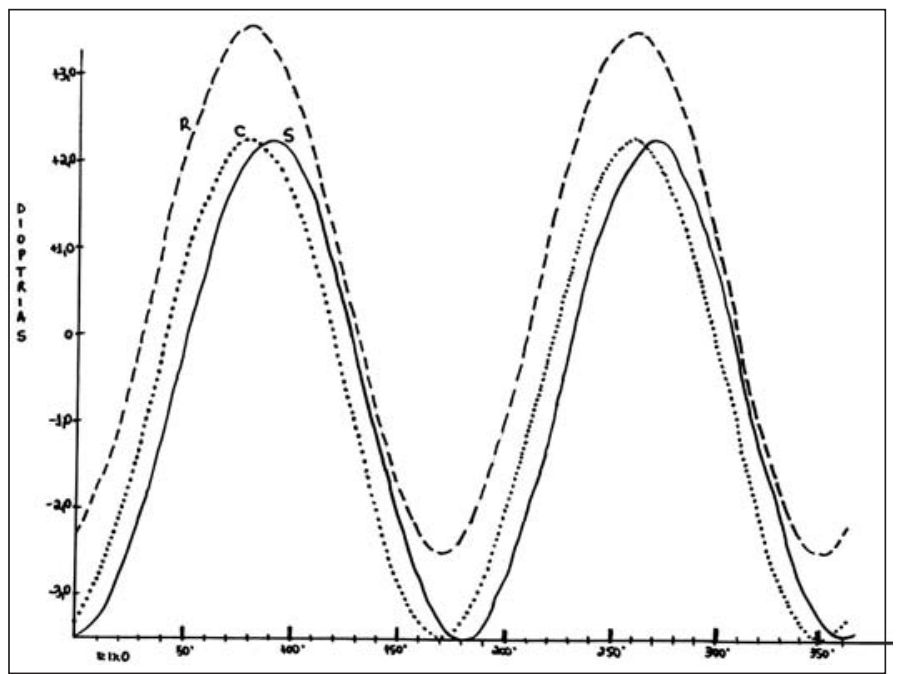

Figura 5 - Diferença entre as curvas $S$ (valores dióptricos da correção óptica nos diferentes meridianos -ou eixos- oculares, com a cabeça ereta) e C (idem, com a cabeça apropriadamente inclinada). Curva da correção ideal (R) 
astigmatismo hipermetrópico simples de $+2,00$ D ou uma esférica de -1,00 D (convertendo-o num hipermétrope simples de $+1,00 \mathrm{D}$ ). A acomodação de 1,00 D produziria, para o caso de astigmatismo residual, o círculo interfocal na retina (mas sem corrigir a distorção astigmática) enquanto para a hipermetropia pura (esférica) induzida, uma correção absoluta seria obtida. A figura 6 ilustra claramente essas diferenças e suas variações.

São várias as causas de $\mathrm{PVC}^{(6)} \mathrm{e}$, em oftalmologia, compensações de estrabismo, ou bloqueio de nistagmo são as mais freqüentes. No entanto, alterações mais simples como erros refracionais mal corrigidos também podem gerar PVC e, muitas vezes, passar despercebidos. Refratometria sob cicloplegia e teste de lentes são fundamentais para um diagnóstico preciso, devendo ser complementados pela ceratometria para se avaliar a posição do eixo do cilindro nos pacientes que não possibilitam a refratometria subjetiva, uma vez que a grande maioria dos grandes astigmatismos é de face anterior da córnea.

\section{ABSTRACT}

Abnormal head position is a compensatory condition which improves patients' vision. It can be caused by ophthalmological problems such as oculomotor imbalances (strabismus, nystagmus) and high astigmatisms. However, it results in esthetic impairment, orthopedic trouble and facial asymmetries. We describe a case of a girl, JL, 8 years, with abnormal head position tilted to the left since the last glasses were prescribed. The correction used by the patient was: right eye $=$ $+2.00 \mathrm{sph} \diamond-5.5 \mathrm{cyl} 180^{\circ}$ and left eye $=+2.25 \mathrm{sph} \diamond-5.75 \mathrm{cyl}$ $180^{\circ}$. In tilted position, the correct visual acuity was: right eye $6 / 12$ and left eye 6/9. No deviations were noted by the cover test and the remaining ophthalmological examination was completely normal. Retinoscopy under cycloplegia and subjective test showed right eye $=+3.50 \mathrm{sph} \nabla-6.00 \mathrm{cyl} 10^{\circ}$ and left eye $=+3.50 \mathrm{sph} \nabla-6.00 \mathrm{cyl} 170^{\circ}$, with visual acuity $6 / 6$ in both eyes. With adequate prescription, the head position was normalized. Wrong cylindrical positions for correction of high astigmatisms may cause abnormal head position. Retinoscopy

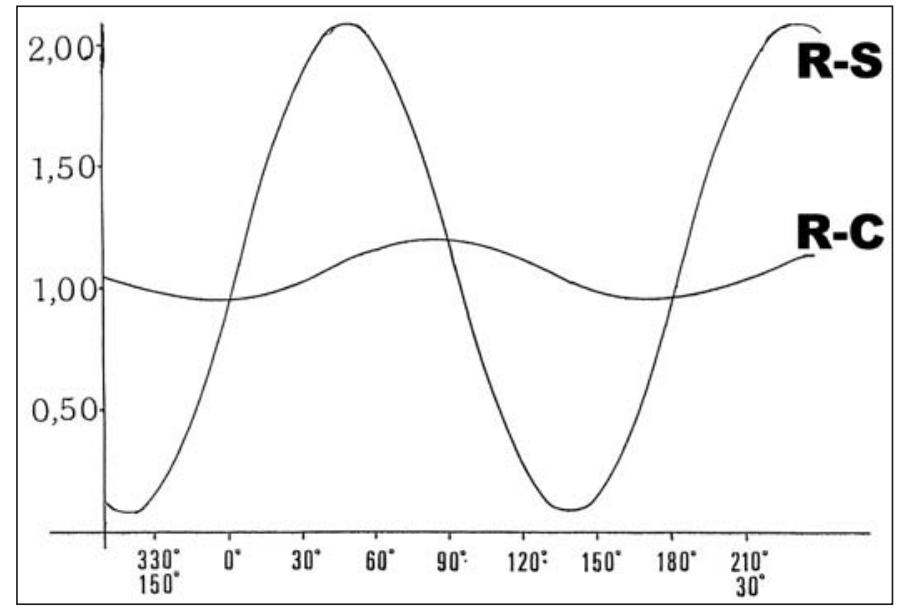

Figura 6 - Valores de hipermetropia residual do olho esquerdo nos vários planos do sistema óptico ocular dados pela diferença entre a refratometria $(R)$ e a correção óptica usada (pela prescrição inadequada), em posição normal da cabeça (S) e com a cabeça inclinada para esquerda (C), isto é, respectivamente valores de R-S e R-C

under cycloplegia and subjective test are essential for precise diagnoses and prescriptions.

Keywords: Head/physiopathology; Posture; Astigmatism; Strabismus; Reflex, vestibulo-ocular/physiology; Nystagmus, pathologic; Oculomotor muscles; Refractometry; Case reports [publication type]

\section{REFERÊNGIAS}

1. Carvalho LEMR. Nistagmo. Arq Bras Oftalmol.1998;61(4):473-5.

2. Souza-Dias C, Díaz JP. Estrabismo. São Paulo: Santos; 2002.

3. Campos EC, Schiavi C, Bellusci C. Surgical management of anomalous head posture because of horizontal gaze palsy or acquired vertical nystagmus. Eye. 2003; 17(5):587-92.

4. Stevens DJ, Hertle RW. Relationships between visual acuity and anomalous head posture in patients with congenital nystagmus. J Pediatr Ophthalmol Strabismus. 2003;40(5):259-64.

5. Jorge AAH. Viscosidade como fator frenador de rotações [tese]. Ribeirão Preto: Faculdade de Medicina de Ribeirão Preto, Universidade de São Paulo; 2003.

6. Barcellos RB, Carvalho LEMR, Souza-Dias CR. Efeito da inclinação da cabeça sobre a acuidade visual de pacientes astigmatas corrigidos com óculos. Arq Bras Oftalmol. 1996;59(1):52-61.

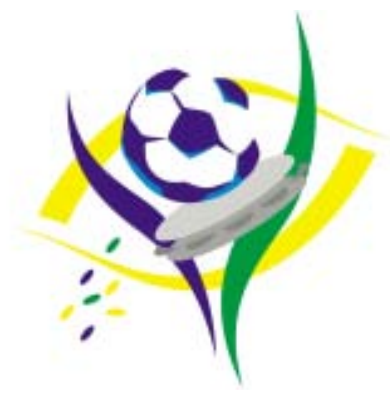

\title{
2006 CONGRESSO MUNDIALDE OFTALMOLOGIA
}

\author{
XXX Congresso Internacional de Oftalmologia \\ XXVI Congresso Pan-Americano de Oftalmologia \\ XVII Congresso Brasileiro de Prevenção da Cegueira
}

São Paulo - Brasil - 19-24 fevereiro 2006 ww w.ophthalmology2006.com.br 